\title{
Best student talk \\ (CLA 2016, University of Calgary) \\ Neil Banerjee (University of Toronto) \\ "Of monsters and modals"
}

In his talk, Banerjee focused on distributional differences between epistemic possibility and necessity modals in English and Kinyarwanda (with regard to temporal shift and the modal base's shift from the speaker to another individual). He argued that epistemic modals involve a context-index split, proposing that under attitude verbs the context is overwritten with an index (due to a "monstrous" operator selected by an attitude verb). His proposal predicts that, cross-linguistically, modals are expected to behave differently in matrix clauses, under attitude verbs and in the consequent of a counterfactual conditional. The judges emphasized Banerjee's strong command of complex theoretical ideas, his effectiveness in linking the data to the formal tools used in his analysis, and the ease in how he handled the question-andanswer period. The judges were also impressed by the breadth of his analysis, which sets the ground for further study of epistemic modals in a cross-linguistic perspective.

\section{Meilleure communication étudiante (ACL 2016, Université de Calgary) Neil Banerjee (Université de Toronto) "Of monsters and modals»}

Dans sa communication, Banerjee a mis l'emphase sur les différences distributionnelles entre les auxiliaires modaux de possibilité et les auxiliaires modaux de nécessité en anglais et en kinyarwanda (plus particulièrement, il s'agit du glissement dans la référence temporelle et dans la base modale relative à l'énonciateur). Selon la thèse de Banerjee, les modaux à valeur épistémique impliquent une division entre contexte et indice. Banerjee propose qu'avec les verbes d'attitude, le contexte est oblitéré par un indice référentiel (dû à un opérateur « monstrueux » sélectionné par 
un verbe d'attitude). Son hypothèse prédit que, dans toutes les langues, les modaux devraient se comporter de façon différente dans la phrase matrice, dans la subordonnée d'un verbe d'attitude, et dans le conséquent d'une conditionnelle contrefactuelle. Les évaluateurs ont souligné que Banerjee a fait montre d'une maîtrise solide de concepts théoriques complexes, d'une bonne capacité à mettre en rapport les données et les outils formels de l'analyse et d'une grande facilité à gérer la période de questions. Les évaluateurs ont également été impressionnés par la portée de l'analyse de Banerjee, qui jette les bases d'études ultérieures portant sur les auxiliaires modaux à valeur épistémique dans une perspective interlinguistique.

\section{Best student poster \\ (CLA 2016, University of Calgary) \\ Malina Radu (University of Toronto) \\ "Conditioned variability in the realization of Romanian rhotics"}

Radu's poster presented a phonetic analysis of Romanian rhotics, with the aim of identifying possible sources of their variability (word-internal position, register, and word type). On the basis of results from two production tasks with 10 native speakers of Romanian, Radu observed different realizations of rhotics extending beyond those previously attested, namely tap and trill variants. The judges unanimously noted that she was extremely comfortable talking about the motivation and implications of her analysis, even if this information was not on the poster. In fact, Radu's research is part of a larger project that examines the acquisition of Spanish by Romanian speakers and the realization of rhotics in both languages. She very clearly had a sense of this larger research program and of how her poster presentation fits in. Finally, she was open to suggestions and ideas, and she was able to answer questions that went beyond what was shown on the poster. 


\section{Meilleure affiche étudiante (ACL 2016, Université de Calgary) Malina Radu (Université de Toronto) "Conditioned variability in the realization of Romanian rhotics»}

L'affiche présentée par Radu proposait une analyse phonétique des consonnes rhotiques du roumain, dont le but était d'identifier les sources possibles de la variabilité (position interne au mot, registre et type de mot) dans ces consonnes. À partir de deux tâches de production réalisées par 10 locuteurs natifs de roumain, Radu a observé différentes réalisations des consonnes rhotiques allant au-delà des variantes attestées antérieurement, c'est-à-dire la vibrante battue et la vibrante roulée. Les évaluateurs ont noté de façon unanime que Radu était tout à fait à l'aise quand il s'agissait d'expliquer les fondements et les implications de son analyse, même si ces informations n'apparaissaient pas sur son affiche. De fait, la présentation de Radu fait partie d'un projet plus vaste qui examine l'acquisition de l'espagnol par des locuteurs de roumain et la réalisation des consonnes rhotiques dans ces deux langues. Radu a clairement expliqué comment son analyse s'inscrit dans ce programme de recherche. De plus, elle a su démontrer de l'intérêt face aux suggestions et idées qui lui ont été communiquées, et elle a pu répondre à des questions qui dépassaient largement l'analyse présentée sur son affiche. 